\title{
Wireless Underground Sensor Network for Monitoring Various Fields Using Magnetic Induction
}

\author{
P. Manigandan ${ }^{\mathrm{a}, 1}$, C. Balaji ${ }^{\mathrm{b}}$, M. Ramanan ${ }^{\mathrm{b}}$, S. Ragul $^{\mathrm{b}}$ \\ ${ }^{a}$ Assistant Professor, Department of EEE, SRM TRP Engineering College, Trichy, TN, \\ India \\ ${ }^{b} U G$ Student, Department of EEE, SRM TRP Engineering College, Trichy, TN, India
}

\begin{abstract}
The frequent accidents of mining safety caused severe losses and massive cost losses. The global mining sector urgently needs to improve operational efficiency and the overall safety of mines. This article suggests a WUSN based mining safety monitoring. The monitoring system collects temperature, moisture, soil vibration and gas values around the mine, and then transmits the data through wireless underground sensor network. Here Transmission based on magnetic induction (MI) is being suggested, in this approach soil is medium for communication so based on the soil conductivity the measured information are transferred. It is also an early warning system, which will help all miners in the mine to save their lives before a victim happens.
\end{abstract}

Keywords: Magnetic Induction, WSUN

\section{Introduction}

India is a big nation with abundant coal reserves. However, the actual healthy coal mine output level remains low, and disasters in coal mines have become more common in recent years, resulting in significant loss of property and life. Coal mine protection issues have increasingly risen to the forefront of the nation's and society's concerns. Because of the complexities of the mining setting and the wide range of coal mine job conditions [1], it is important to keep a close eye on the mine operating environment. Monitoring systems for traditional coal mines are typically wired systems for the network that take a significant role in ensuring mine of coal safety. As the mining areas and the depth of coal mines are expanding continuously, a lot of paths have become blind and many concealed hazards. Furthermore, laying wires, which is costly and time- consuming in the coal mines.

A coal mine Safety Monitoring System will be designed on the basis of an underground Wireless Sensor Network (WUSN), to improve production safety monitoring and reduce coal mining casualties [2]. Wireless sensor networks consist of a large number of small volume and low cost micro sensor nodes. It has self-organized wireless communication capabilities. 
Underground mines are typically large labyrinths with long, narrow tunnels measuring a length of kilometers and width of several meters. Thousands of miners are required to operate under extreme conditions, and hundreds of miners die each year from mining accidents. Underground mining activities are now generally accepted as posing a high danger. In order to ensure mining safety and to manage various tasks, a monitoring and controlling system must be installed as a critical infrastructure. Underground coal mining often consists of random corridors and branch tunnels which make it extremely difficult to deploy a network skeleton.

In such cases it may take particular advantages for the automation of underground monitoring and control thanks to quick and flexible use of the wireless underground sensor network (WUSN) and any other sensing devices. Moreover, the multi-hop method of transmission can be adapted well to the tunnel structure and can thus provide a sufficient scalability to construct a mining system and is very suitable for extensive monitoring and control in the coal mineral industry. Mine detection methods have become more sophisticated as technology has advanced, but accidents in underground coal mines continue to occur. Coal mine Safety has increasingly become a major source of concern for society and the nation. The harsh climate and variable working conditions in coal mines are the primary causes of disasters. As a result, mine control systems must be implemented for the sake of protection. Traditional coal mining used to be a trend for wired network networks, which have played a major role in ensuring secure output in coal mines [3]. The continuous extension and deep expansion of the operating areas have turned into blind areas where numerous unnoticed hazards are concealed. Furthermore, costly cables cannot be laid there, that also takes time. Consequently, to ensure a good performance while protecting the environment, it is essential to have a wireless sensor network mine monitoring system installed in these mines.

\section{Related Work}

For transmitting the calculated parameters, the current device uses a zigbee network. In Figure 1. Show this system the measures the parameters like temperature sensor and moisture sensor the measured sensor details are transferred based on zigbee network [4] . The received details are displayed on LCD. Traditional wireless signal transmission technology using EM waves can be used in many realistic cases only at very small distances due to high trajectory loss and vulnerability to soil moisture changes.

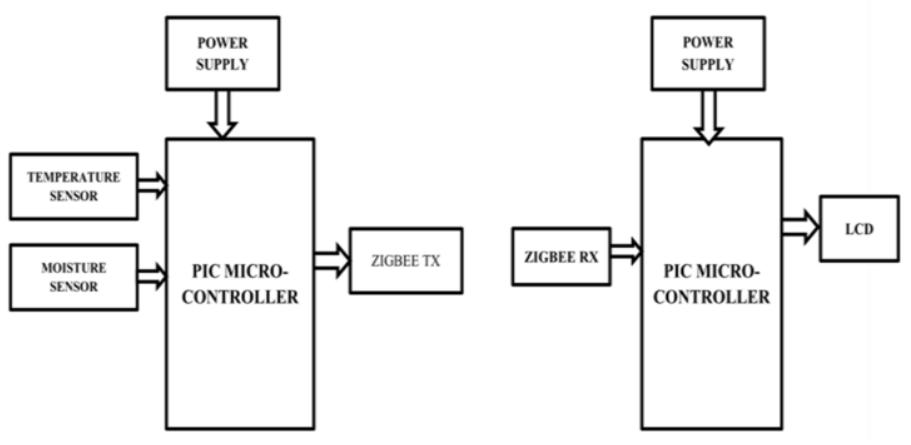

Figure 1. Related Work block diagram 
In wireless signal propagation techniques with electromagnetic (EM) waves, only very short transmission can be implemented.

\section{Methodology System}

Continuous monitoring is needed, which necessitates the use of a reliable and precise sensor system. Various techniques are employed for the detection of these toxic gases, including the use of a gas sensor type semiconductor. These sensors can be used in coal mines, but they can also cause problems in the mining process. The sensor system was often damaged by accident. Another method is to employ a robot. These robots are effective, but they come at a high price. However, there is another way to obtain an efficient and low-cost sensor implantation solution [5] ; the prediction of explosion with the use of sensors and microcontrollers is the secret to manage coal mining accidents, as well as the generation of an alarm device before critical atmospheric levels are reached [6] in shown in Figure 2.The secret to preventing coal mine accidents is to anticipate outbursts using sensors and microcontrollers, as well as to set up a warning system before critical atmospheric levels are reached. Continuous monitoring is needed, which necessitates the use of a reliable and accurate sensing device. Proposed system consists of temperature sensor, Pressure sensor, vibration sensor and gas sensor for monitoring the parameter like temperature, Pressure condition, vibration level and gas. The measured parameters are transferred WUSN. At receiver side, it receives the measured parameters display on LCD and Buzzer is used for the purpose of emergency indication.

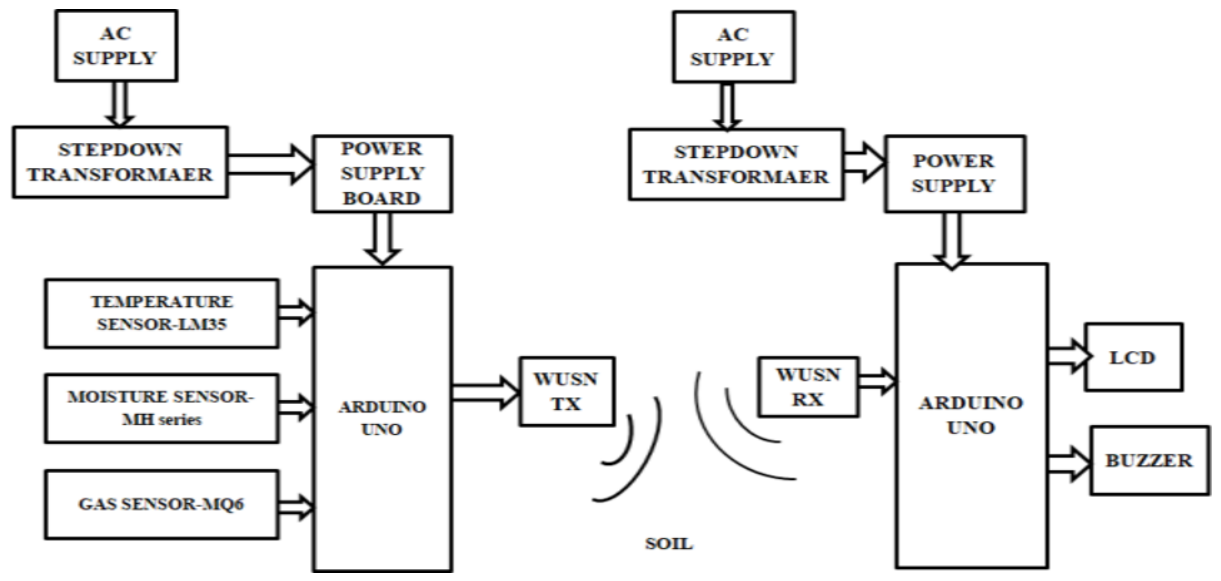

Figure 2. Proposed block diagram

In WUSN transmitter side temperature sensor, moisture sensor and gas sensor are interfaced with the Arduino Uno micro controller [7-9]. It picks up the sensor data that has been tested. Based on a magnetic induction approach, the measured sensor information were transmitted using the WSUN transmitter module. At receiver side the WUSN receiver module receives the information and it displayed on the LCD. If the measured sensor details goes high means buzzeris used to indicate It is highly efficient because it decreases the signal propagation vulnerability in properties of the soil, reduces the track loss and is easy to use Easy to install. 


\section{WUNS Module}

Sensor nodes are linked to the wireless underground sensor network, and are randomly distributed in the environment to monitor personnel locations or other interesting events. Here sensor nodes randomly distributed to occupy all corners of the environment as it is applied to grid based topology. For the network under consideration (underground sensor network), it is important to know the distance between pairs of nodes as it will allow us to give priority to either distance or energy consumed by nodes in the network [10]. WUSN devices are widely implemented under the ground and no wired connections are required. Each unit contains all sensors, memory, a processor, a radio, a power source and an antenna. Wireless underground sensor networks (WUSN) are made up of wirelessly linked underground sensor nodes that communicate with each other via the soil, and they have the potential to affect a wide range of new applications.

In this basic WUSN circuit as shown in Figure 3, the resistor R1 and the $\mathrm{c} 1$ form one adjustable arm of a simple resistance bridge network. While the two fixed resistors R2 and R3 form the other arm. Both these are connected to amplifier IC. The end of R3 connected to R4 which is connected to com port of controller.

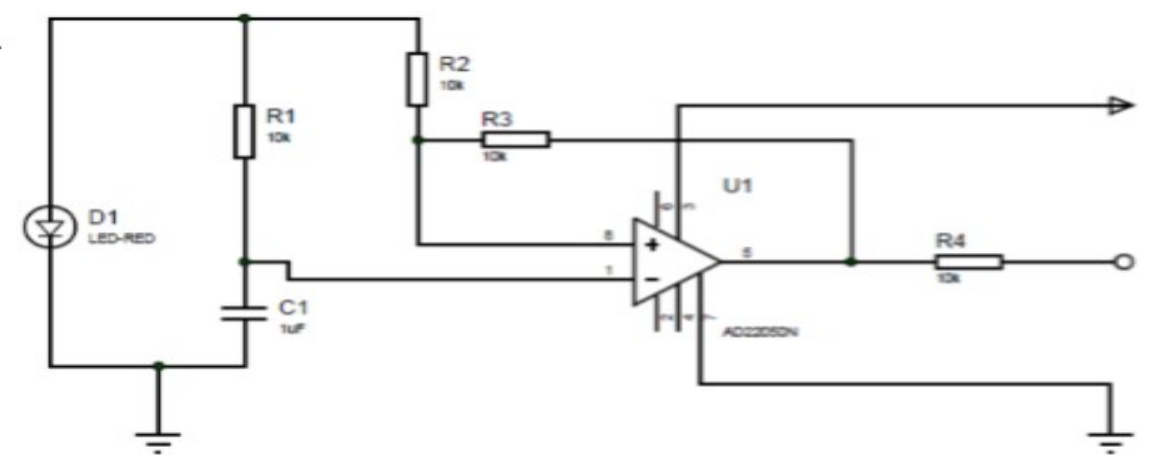

Figure 3. Circuit diagram of WUSN module.

\section{Proteus ISIS7 Simulator}

As a fully functional procedural programming language, Simone Zanella developed Proteus (TEXT Easy to USE Processor) in 1998. Proteus integrates many functions derived from a variety of other languages, including C, BASIC, Assembly, and Clipper/dBase; it is particularly flexible when working with strings, with hundreds of dedicated functions, making it one of the most powerful text manipulation languages is shown in Figure 3. Proteus is named after Proteus, a Greek sea god who looked after Neptune's entourage and provided responses; he was known for his ability to turn into various forms [11]. This language's main aim is to translate data from one type to another. Proteus began as a multiplatform (DOS, Windows, UNIX) system utility for manipulating text and binary files as well as writing CGI scripts. In the Figure 4 the language was later extended to include hundreds of specialist network and serial communication functions, database interrogation, system service design, console applications, keyboard emulation, and ISAPI scripting (for IIS). Although a Linux version is still available, most of these features can only be found in the Windows 
interpreter version. Proteus was created to be useful (simple to use, effective, and comprehensive), readable, and reliable.

\section{Prototype}

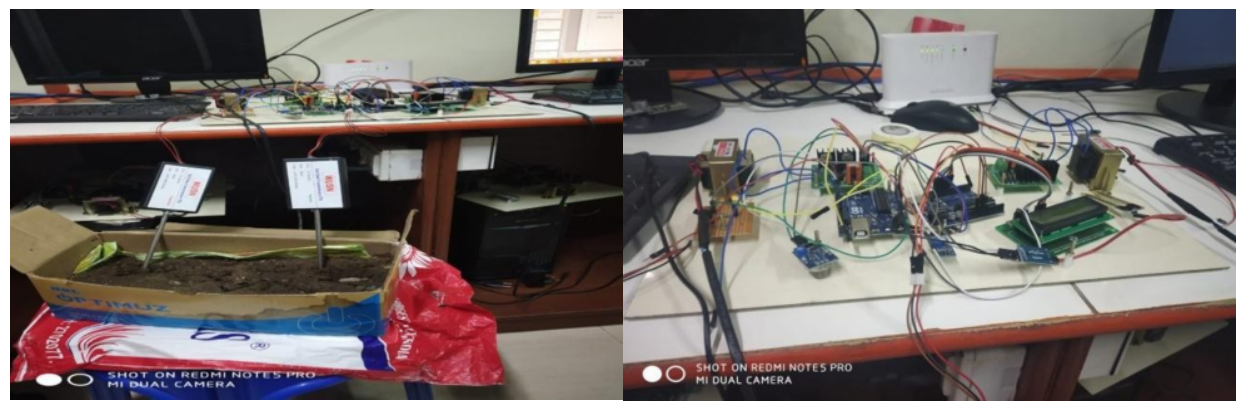

(a)

(b)

Figure 4. (a) Soil Testing system (b), Controlling and monitoring Hub

\section{Conclusion}

The coalmines safety monitoring system is therefore implemented through the use of the wireless network of underground sensors (WUSN). The advances are linked to different aspects of wireless communication and networking based on the magnetic induction approach. Moisture, Gas and temperature level is always sensed and if changes in those values from threshold level then it monitored by the monitoring center. From this proposed we can able to give immediate treatment for workers in case of emergency situation.

\section{References}

[1] Akyildiz, I. F., Su, W., Sankarasubramaniam, Y., \& Cayirci, E. (2002). Wireless sensor networks: a survey. Computer Networks, 38(4), 393-422. https://doi.org/10.1016/S1389-1286(01)00302-4

[2] Matin, M. A., \& Islam, M. M. (2012). Overview of Wireless Sensor Network. In Wireless Sensor Networks - Technology and Protocols. InTech. https://doi.org/10.5772/49376

[3] Akyildiz, I. F., \& Stuntebeck, E. P. (2006). Wireless underground sensor networks: Research challenges. Ad Hoc Networks, 4(6), 669-686. https://doi.org/10.1016/j.adhoc.2006.04.003

[4] Vuran, M. C., \& Silva, A. R. (2010). Communication Through Soil in Wireless Underground Sensor Networks - Theory and Practice (pp. 309-347). https://doi.org/10.1007/978-3-642-01341-6_12

[5] Bansal, R. (2004). Near-field magnetic communication. IEEE Antennas and Propagation Magazine, 46(2), 114-115. https://doi.org/10.1109/MAP.2004.1305555

[6] Karalis, A., Joannopoulos, J. D., \& Soljačić, M. (2008). Efficient wireless non-radiative mid-range energy transfer. Annals of Physics, 323(1), 34-48. https://doi.org/10.1016/j.aop.2007.04.017

[7] Zhi Sun, \& Akyildiz, I. F. (2010). Magnetic Induction Communications for Wireless Underground Sensor Networks. IEEE Transactions on Antennas and Propagation, 58(7), 2426-2435. https://doi.org/10.1109/TAP.2010.2048858

[8] Sheth, A., Toyama, K., Tejaswi, K., Mehta, P., Parekh, C., Bansal, R., Merchant, S., Singh, T., Desai, U. B., \& Thekkath, C. A. (2005). SenSlide. Proceedings of the 3rd International Conference on Embedded Networked Sensor Systems - SenSys '05, 280. https://doi.org/10.1145/1098918.1098954

[9] Werner-Allen, G., Lorincz, K., Ruiz, M., Marcillo, O., Johnson, J., Lees, J., \& Welsh, M. (2006). Deploying a wireless sensor network on an active volcano. IEEE Internet Computing, 10(2), 18-25. https://doi.org/10.1109/MIC.2006.26

[10] Akyildiz, I. F., \& Vuran, M. C. (2010). Wireless Sensor Networks. John Wiley \& Sons, Ltd. https://doi.org/10.1002/9780470515181 